\title{
Partially Coherent Backscatter in Radar Observations of Precipitation
}

\author{
A. R. JAMESON \\ RJH Scientific, Inc., El Cajon, California \\ A. B. KOSTINSKI \\ Michigan Technological University, Houghton, Michigan
}

(Manuscript received 28 September 2009, in final form 23 December 2009)

\begin{abstract}
Classical radar theory only considers incoherent backscatter from precipitation. Can precipitation generate coherent scatter as well? Until now, the accepted answer has been no, because hydrometeors are distributed sparsely in space (relative to radar wavelength) so that the continuum assumption used to explain coherent scatter in clear air and clouds does not hold.

In this work, a theory for a different mechanism is presented. The apparent existence of the proposed mechanism is then illustrated in both rain and snow. A new power spectrum $Z(f)$, the Fourier transform of the time series of the radar backscattered reflectivities, reveals statistically significant frequencies $f$ of periodic components that cannot be ascribed to incoherent scatter. It is shown that removing those significant $f$ s from $Z(f)$ at lower frequencies greatly reduces the temporal coherency. These lower frequencies, then, are associated with the increased temporal coherency. It is also shown that these $f \mathrm{~s}$ are also directly linked to the Doppler spectral peaks through integer multiples of one-half the radar wavelength, characteristic of Bragg scatter. Thus, the enhanced temporal coherency is directly related to the presence of coherent scatter in agreement with theory.

Moreover, the normalized backscattered power spectrum $Z(f)$ permits the estimation of the fractional coherent power contribution to the total power, even for an incoherent radar. Analyses of approximately 26000 one-second $Z(f)$ in both rain and snow reveal that the coherent scatter is pervasive in these data. These findings present a challenge to the usual assumption that the scatter of radar waves from precipitation is always incoherent and to interpretations of backscattered power based on this assumption.
\end{abstract}

\section{Introduction}

Because radars first detected signals backscattered from storms, scientists have been trying to interpret them quantitatively. The earliest breakthrough came when the approach of Rayleigh's (1945) treatment of the scatter of sound waves was applied to the scattering of microwaves by precipitation (e.g., Marshall and Hitschfeld 1953). An essential characteristic responsible for the apparent success of this theory is that the scattering by each particle is incoherent (i.e., independent of all the other scatterers). However, there are now reasons to question the general validity that all backscatter from precipitation must always be incoherent. Is it possible, then, that backscatter by precipitation can sometimes be partially coherent? What does this mean?

Corresponding author address: A. R. Jameson, 5625 N. 32nd St., Arlington, VA 22207-1560.

E-mail: arjatrjhsci@verizon.net
The concept of "coherence" plays a central role in modern physical science. It is multifaceted because there can be spatial coherence, temporal coherence, ensemble coherence, partial coherence, and others [e.g., Wolf (2007) or Ishimaru (1997, p. 78), where coherent field is simply defined as the ensemble average one]. In radar meteorology, coherence is often used to denote different concepts. For example, Doppler radar is termed coherent, but it is "looking" at incoherent targets (precipitation).

Consider, for example, the definition in the first textbook on radar meteorology, Battan (1973, p. 33):

A target composed of distributed targets which move with respect to one another is said to be incoherent. A solid object, such as a metal sphere, would be regarded as a coherent target.

By this commonly accepted definition, precipitation always produces incoherent scatter simply because its constituents move with respect to each other. However, what about partial coherence? Part of our motivation for 
the research described herein stems from the feeling that the previous definition is unduly restrictive. Why? Because precipitation is not perfectly random but has spatial texture. Any such texture can be regarded as a superposition of "spatial gratings" of different strengths and wavelengths. Albeit fleeting, such spatially coherent patterns can resonate with the radar wavelength and produce spatially coherent backscatter, similar to laser speckle in optics (another example is X-ray scattering by amorphous solids: although crystalline solids have a well-defined Bragg scatter structure, the X-ray scatter by amorphous solids is less well defined; however, X-ray scatter is widely used, nevertheless, for material analysis). In radar meteorology, the situation is even more subtle as the "amorphous Bragg scatter" changes with time. However, let us elaborate on the spatial texture of precipitation first.

Previous research has shown that cloud and precipitation are not perfectly random as an ideal gas but rather possess texture: spatial correlations between particle positions. This has a variety of causes. For example, raindrop breakup forms clusters of fragments. Patchiness of cloud particles is caused by the turbulent air, in which these particles are immersed: that is, the formation of patches and filaments is due to the interplay of intense and spotty random vorticity and drop inertia as they fall through the eddies while being partially entrained by them.

As mentioned earlier, such structures imply spatial correlations that are conveniently characterized using the pair correlation function $\eta$ (see appendix A). We note that perfect randomness means that the pair correlation function vanishes at all scales. This is a rather stringent condition; as with any perfection, perfect randomness is difficult to attain. Because of the WienerKhintchine theorem, the existence of Fourier spectral components and hence the presence of spatial periodicities is implied whenever there is a deviation from perfect randomness on some scales.

Our main motivation then is the notion of spatially periodic (albeit fleeting) elements present in precipitation and capable of backscattering in spatially coherent diffraction-like patterns. Although radar returns are still incoherent by the Battan definition because raindrops move with respect to one another, spatial coherence may nevertheless be out there. How do we detect it?

To that end, we ask the reader to consider a periodic spatial pattern of intensities produced by a distant diffraction grating. Then imagine an observer at a point, moving with a constant velocity across such a pattern. Clearly, the observer will detect time-periodic intensity oscillations. Now, let us next choose a frame of reference that moves with the observer. In this case, the observer (analogous to our radar) is stationary, but the distant diffraction grating is in motion. The detected signal, however, will still be time periodic. This simple gedanken experiment suggests that, if we allow some precipitation to be spatially correlated with all the elements of the gratings moving at the same Doppler velocity (see appendix A), then that precipitation may act like diffraction gratings (albeit fleeting at times) thereby producing time-periodic radar echoes. However, detection is a difficult task because the temporal periodicity is eventually destroyed by reshuffling. Furthermore, despite the coherence, the usual in-phase and quadrature statistics of the real and imaginary components of the complex amplitudes ( $I$ and $Q$ ) still hold as our gratings ("superdrop" elements) obey the same rules as the raindrops themselves (e.g., they move around, reshuffle, and scatter independently). In fact, even when an airplane goes through the radar resolution volume, $I$ and $Q$ statistics still remain Gaussian (illustrated later in Fig. 10). Thus, would the statistics of $I$ and $Q$ alone suggest that a moving airplane is an incoherent target? This is just another illustration of the difficulty with the notion of coherence.

Returning to our spatial periodicities, however, if these gratings reshuffle more slowly than the raindrops themselves, the backscatter may be proportional to $N^{2}$ rather than $N$, the number of raindrops in a sample volume. The main goal of this research is to present evidence for the coherent component in radar backscatter. Admittedly, the separate items presented later may not seem conclusive; however, the totality of evidence and the variety of "symptoms" present for rain, snow, and rain-airplane combinations of these pieces deliver a compelling picture. The symptoms of partially coherent scatter may be as follows:

Time periodicity may have symptoms of Bragg scatter by having maxima associated with multiples of halfinteger radar wavelength.

The periodic structure in precipitation, because of spatial extent, may take longer to reshuffle. In other words, signal coherence in time as evidenced by increased coherence time reflects structural coherence in space.

These conditions are presented formally in appendix A.

Currently, however, incoherent scatter is assumed by some to be all that there is. The concept of incoherent scatter has an interesting history in radar meteorology extending all the way back to the work on sound by Rayleigh (1945) in 1871. In particular we quote from Rughaven (2003, p. 17):

If we assume that the scattering is incoherent, i.e. the particles are randomly placed and the phases of the echoes from individual scatterers are distributed over an interval $2 \pi$, the total back scatter cross section is the sum of the individual cross sections. 
The author is completely honest in that the key word here is "assume," because the validity of the assumption of incoherent scatter has yet to be proven. In fact, if one returns to the earliest work on radar scattering, the possibility of coherent scatter is recognized and developed (Siegert and Goldstein 1990) where, because of the spatial structures in precipitation, " . . . a certain amount of coherent scatter can be expected."

The difficulty was and still is that there was never a way to determine how much of the scatter by precipitation was coherent. Consequently, over time the second term in Eq. (15) in Siegert and Goldstein (1990) was simply ignored, and it became that mantra in the field that all scatter was incoherent. This approach was reinforced by two other factors. The first factor is the apparent success in describing the observed signal fluctuations (e.g., Marshall and Hitschfeld 1953; Lhermitte and Kessler 1966) developed assuming the scatter was incoherent. We show here, however, that classical signal statistics cannot be used to disprove the presence of coherent scatter, because the coherent scatterers act like superdrops moving in the wind just like any other scatterer. Consequently, the signal statistics remain unaltered (some argue that the statistics should be Ricean, but that is incorrect as we discuss later in the paper). The second factor is the resurrection of the work of Siegert and Goldstein by Gossard and Strauch (1983), as we discuss next.

Coherent scatter is not new to atmospheric measurements by radar, of course. Following the general formalism of Tatarskii (1961), signs of coherent scatter in clear air have been interpreted in terms of the index of refraction fluctuations caused by the turbulent energy cascade (e.g., Gossard and Strauch 1983). Some investigators have extended this approach to explain (e.g., Erkelens et al. 2001) apparent radar coherent scatter in clouds (see Knight and Miller 1993) and smoke (Rogers and Brown 1997) by treating the particles as a continuum in which the inhomogeneities in the spatial concentration of the droplets are equivalent to fluctuations in the index of refraction occurring on the appropriate Kolmogorov turbulent scales. As Gossard and Strauch (1983) point out, however, such an approach cannot produce coherent scatter in precipitation, because hydrometeors are distributed too sparsely in space (relative to radar wavelength) for the continuum assumption to hold. However, Kostinski and Jameson (2000) suggested a different mechanism.

The theory for this alternative mechanism for the generation of coherent scatter from precipitation is presented in appendix A. This approach requires neither the continuum assumption nor Kolmogorov turbulent scaling, and it incorporates the effect of velocities ignored by Gossard and Strauch (1983). However, it does require both temporal coherency and spatial coherency; that is, radar coherent backscatter is possible when elements of a structure all move at nearly the same Doppler velocity over at least a brief interval and when the elements of the spatially correlated structures of precipitation are in resonance with the radar wavelength. In that case,

$$
\begin{aligned}
\langle I\rangle & =\left\langle\sum_{i} a_{i}^{2}\right\rangle+\left\langle I_{B}(t)\right\rangle \\
& =\bar{N} \overline{a^{2}}+\bar{N}^{2} \overline{a^{2}}\left\langle F_{B}(t)\right\rangle \\
& =I_{\text {incoherent }}+I_{\text {coherent }}
\end{aligned}
$$

where $\langle\cdot\rangle$ represents the time average over an ensemble of observations, and $F_{B}$ given by

$$
F_{B}(t)=\frac{2 \pi}{V k} \int_{0}^{\infty} \ln (l) \sin (-2 k l) d l,
$$

where $l$ is the separation distance between scatterers in the direction of the transmission, $\eta$ measures the correlation in the number of drops between disjoint elemental volumes separated by $l, k$ is the wavenumber, $\overline{a^{2}}$ is the mean squared scattered amplitude, and $\bar{N}$ is the mean total number of particles in sample volume $V$. Note, too, that the incoherent part goes as $\bar{N}$ while $I_{B} \propto \bar{N}^{2}$ (see appendix A). The remainder of this paper is devoted to illustrating this mechanism in both rain and snow.

In the next section, we present several independent lines of evidence, all of which point toward the presence of coherent scatter. We begin with the most direct evidence first.

\section{Observations}

In this section, radar data in both rain and snow were collected using the National Science Foundation Colorado State University-University of Chicago-Illinois State Water Survey (CSU-CHILL) radar facility at Greeley, Colorado, which is operated by the Colorado State University. This radar has a $1.1^{\circ}$ beamwidth. It operates at a frequency of $2.725 \mathrm{GHz}$, corresponding to a nominal wavelength of $11.01 \mathrm{~cm}$. Time-series observations of the complex backscattered amplitudes ( $I-Q$ pairs) were collected holding the antenna stationary, 1024 times per second at vertical polarization. [Note that the analyses presented below will not function for a moving antenna because such motion, which is not considered here, injects non-Rayleigh signal statistics (see Jameson and Kostinski 1996) into the problem. Coherent scatter is still present, but it is then not measurable using these techniques.] In the rain, observations were collected over 332 bins of 150-m range over a distance of about $3-53 \mathrm{~km}$ from the radar. The elevation angle was $1.82^{\circ}$ so that the bottom of the main lobe of the beam was around $600 \mathrm{~m}$ above the surface at about $30-\mathrm{km}$ range. These measurements are through weak convection containing 
a few convective cores. Likewise, observations were gathered in snow over 218 bins of 150-m range over a distance of about $3.30-36 \mathrm{~km}$ from the radar. The elevation angle was $2.54^{\circ}$ so that the bottom of the main lobe of the beam was around $700 \mathrm{~m}$ above the surface at about a range of $20 \mathrm{~km}$.

Throughout this paper, the analyses focus largely on the radar backscattered intensities $Z$ with little reference to the Doppler information. One of the important exceptions is that the 1000-point Doppler spectra were calculated and then used to compute the observed standard deviations of the velocities $\sigma_{v}$. These, in turn, were used to compute the expected 1/e times to decorrelation (where $e$ is Euler's number) using standard formulas devised assuming that the Doppler spectra were Gaussian [the decorrelation time is simply the time (pulse to pulse lags) it takes for the complex amplitudes-powers to become statistically independent because of particle relative motions]; that is, using the relation (6.24) in Atlas (1964, and many other references), it is argued that

$$
\tau_{1 / e}=\frac{0.796 \lambda}{\sigma_{v}}
$$

where $\lambda$ is the radar wavelength in centimeters, $\sigma_{v}$ is in meters per second, and $\tau$ is in milliseconds.

The reason for using the 1/e time to decorrelation is that, unlike the time to 0.01 decorrelation, it is relatively easy to measure directly from the complex autocorrelation function magnitudes $\rho$ of the of the complex amplitudes independent of (3). For incoherent scatter and for approximately Gaussian Doppler spectra (an assumption made throughout the radar meteorology literature and, e.g., one of the primary justifications for pulse pair processing for Doppler velocity information), the values for $\tau$ computed from the Doppler velocity standard deviations and those directly measured using $\rho$ should be quite similar. Surprisingly, that is not what Fig. 1 shows.

With a range of the observed standard deviations of the Doppler velocities approximately up to a few meters per second in both the snow and the rain, one would expect the usual 1/e decorrelation times of around $4-8 \mathrm{~ms}$ at the most. Although 5-6 ms are the mean and peak frequency values in Fig. 1 observed directly in the rain, the peak calculated using $\sigma_{v}$ is only about $3 \mathrm{~m} \mathrm{~s}^{-1}$ (70\% of the calculated $\tau$ values are $\leq 3 \mathrm{~ms}$ ). Moreover, $45 \%$ of the observed values are larger than $5 \mathrm{~ms}$ with $5 \%$ of the values $\geq 10 \mathrm{~ms}$. The snow is even more remarkable with a peak in the histogram frequency (Fig. 1b) of the directly observed values of $20 \mathrm{~ms}$ and a mean of about $21 \mathrm{~ms}$; however, the mean value derived using $\sigma_{v}$ is only $4 \mathrm{~m} \mathrm{~s}^{-1}$ (again, $70 \%$ of the calculated $\tau$ values are $\leq 4 \mathrm{~ms}$ ).

Furthermore, $30 \%$ of the observed values occur at $\tau \geq 25 \mathrm{~ms}$. These $\tau$ values are much, much larger than
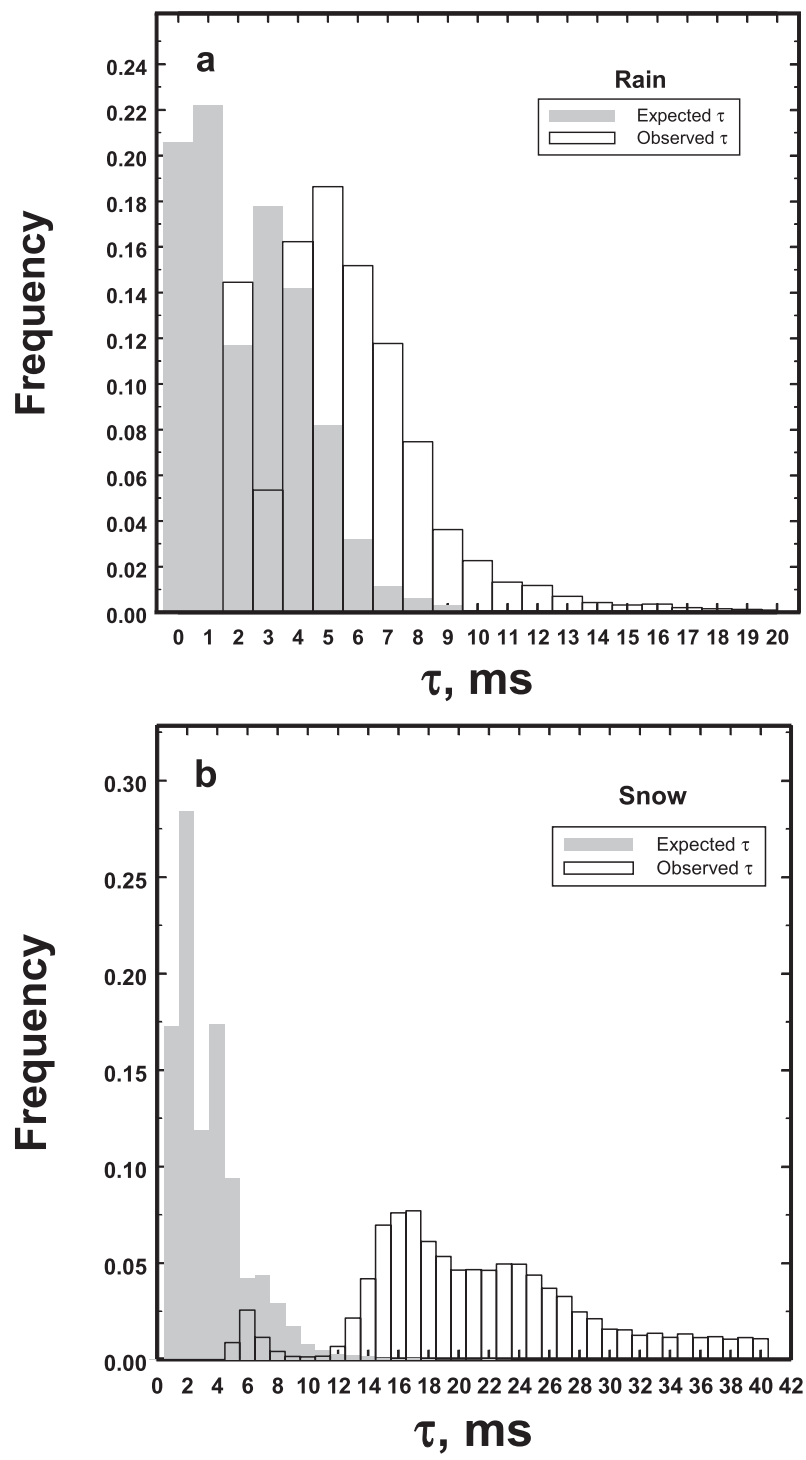

FIG. 1. The histograms of the 1/e times to decorrelation for (a) 15600 samples in the rain and (b) 10400 samples in the snow. The expected values of $\tau$ are calculated using the observed standard deviations of the Doppler velocities. The excess observed correlation in both rain and snow indicates the presence of an additional source of coherence.

one would expect for the traditional, incoherent scatter decorrelation. For example, in the snow at range bin (RB) 131 between 28 and $29 \mathrm{~s}$, the observed standard deviation of the velocity was $1.50 \mathrm{~m} \mathrm{~s}^{-1}$. According to classical theory, this implies $1 / e$ decorrelation time of about $6 \mathrm{~ms}$; however, the observed value was $16 \mathrm{~ms}$. Clearly, the observed large values cannot be used as a measure of the time to decorrelation for the incoherent component.

More importantly, why are these decorrelation times so much larger than particle reshuffling would imply? What is the origin of the extra coherence evident in both 


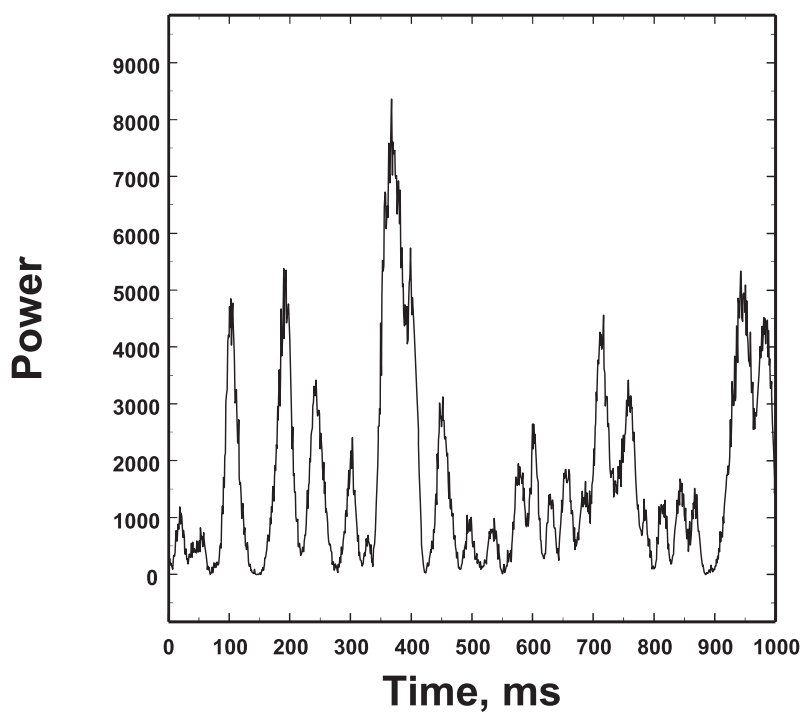

FIG. 2. The time series of the radar-backscattered intensity measured in snow at RB 131 that exhibits clear and striking periodicity.

the rain and the snow observations? These values would be easy to understand if a coherent target such as an airplane was in every sample volume. The coherency of the airplane would greatly extend the observed time to decorrelation of any precipitation. However, aside from the absurdity of finding a coherent target in every observation, potential ground clutter and other targets (such as airplanes) were easily identified and removed from further analyses as discussed in appendix C. These differences are real and perplexing. Is there another source of coherency? As will be shown, the answer appears to be yes.

One clue to the origin of added coherency appears in Fig. 2 using observations in range bin 131 in the snow. The periodicities of the oscillations in $Z$ are readily apparent. For completely incoherent scatter having no correlation, one would expect random spikes. Incoherent data, of course, can be correlated as just discussed. However, although such correlation can smooth over some of the spikes by effectively bunching similar data together, there is little reason to expect such correlation alone to generate what appears to be some striking periodicities in Fig. 2.

To study the spectral components of these modulations we take the Fourier transform of the radar backscattered power $Z(f)$ normalized by the total power as illustrated in Fig. 3 (note that this is not equivalent to the Doppler spectrum, which is the Fourier transform of the backscattered complex amplitudes as discussed at the end of appendix A). The quantity $Z(f)$ is similar to the socalled fluctuation spectrum arising from differential



FIG. 3. An example of the radar backscatter power spectrum $Z(f)$ in snow at RB 131 plotted as a function of frequency. The horizontal line is the threshold used to separate coherent scatter from incoherent scatter noise, as discussed in appendix C. The $Z(f)$ resulting from velocity fluctuations alone is plotted as well. Clearly, the observed $Z(f)$ far exceeds that which can be attributed to velocity fluctuations.

particle velocities (e.g., Atlas 1964, 397-403) where $f$ is the differential frequency for purely incoherent scatterer, but it differs in important ways. Although the fluctuation spectrum is based solely on Doppler information, $Z(f)$ includes non-Doppler information; that is, whenever coherent scatter is present, there is an additional component to $Z(f)$ because the backscattered power can oscillate regardless of any differential velocities. This is important because the differential velocity spectrum can then be calculated independently of the Fourier transform of the reflectivity time series so that the comparison of $Z(f)$ to the fluctuation spectrum can identify those spectral features not associated with differential velocities.

For incoherent scatter alone in which each sample is statistically independent from the others, any frequency can occur but $Z(f)$ will appear nearly flat. In reality, however, there is usually a Doppler spectrum that implies the existence of signal correlation. This, in turn, leads to a "coloration" of $f$ at lower frequencies; that is, the relative powers at different $f$ s increase as $f$ decreases. In spite of this rising incoherent scatter "noise" level as $f$ decreases, Fig. 3 shows that, when coherent scatter is present, the observed powers in the lower $f$ s can significantly exceed what would be expected from differential velocities alone; that is, in Fig. 3 we compute the $Z(f)$ from the observed Doppler velocities and compare it to the Fourier transform of the observed time series of the radar reflectivity. Obviously, the velocities alone cannot 


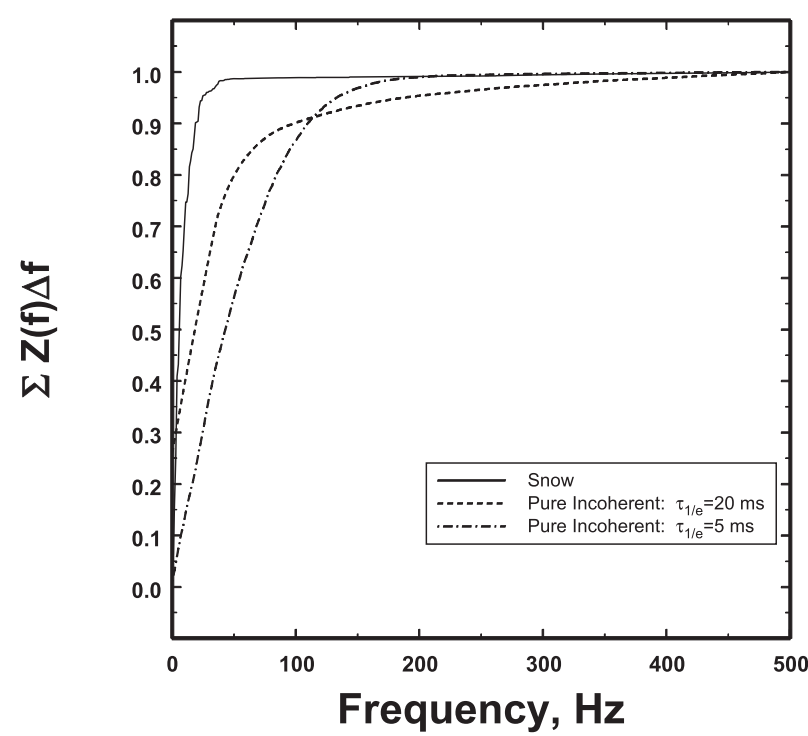

FIG. 4. Plots of the accumulated spectral power $Z(f) \Delta f$ for the snow example and for purely incoherent scatter having the decorrelation time $(5 \mathrm{~ms})$ calculated from the observed standard deviation of the Doppler velocities. Note the much larger contributions of spectral power at frequencies less than about $50 \mathrm{~Hz}$ in the data than occurs for purely incoherent scatter (also see Fig. C2). Even when the observed correlation time of $20 \mathrm{~ms}$ is assigned entirely (but unrealistically) to the incoherent scatter alone, the shape of the incoherent scatter curve still does not approach the observed curve.

explain the significant fluctuations of $Z(f)$ in Fig. 3 . (Because we must take the direct Fourier transform of the time series of reflectivity measurements to see potential coherent scatter, we must then take into account noise that does not appear in the usual methods for calculating the differential velocities fluctuation spectrum; this is addressed using thresholds in appendix C.)

Specifically, in appendix $\mathrm{C}$ it is shown that a spectral power thresholds $T$ can be defined [one for rain (0.018) and one for snow (0.022)] above which incoherent scatter is largely (but not perfectly) excluded. Figure 3 suggests that these thresholds are likely conservative, so that should be kept in mind in the subsequent discussion. Therefore, $Z(f)$ is a new useful radar power spectrum in which the $f \mathrm{~s}$ are now those of the oscillations in $Z$ (and in the magnitudes of the amplitudes) rather than those associated with the usual Doppler velocity power spectrum. However, the integration over $Z(f)$ over all the frequencies gives the total backscattered power just as for the Doppler spectrum.

Thus, in Fig. 3 the presence of statistically significant spectral powers rising well above the threshold and those resulting from velocity-induced oscillation alone can be attributed to the only other kind of scatter there is (see appendix A): namely, coherent scattering. Moreover, $Z(f)$ can be used to estimate the coherent power contribution to the total power or, in our case, the fractional coherent power contribution $\mathfrak{F}$ to the total normalized spectrum as discussed in appendix $\mathrm{C}$.

The difference between $Z(f)$ for data containing a mixture of incoherent and coherent scatter (see appendix A) and those for purely incoherent scatter is shown in Fig. 4 for the data in Fig. 3 but using the accumulated spectral powers $[\Sigma Z(f) \Delta f]$. The upper line is for the snow observations of Figs. 2-3, whereas the lower line corresponds to a $\tau$ calculated using the observed Doppler velocity standard deviation as discussed earlier. This latter time to decorrelation is taken to represent $\tau$ of the incoherent scatterers. Clearly, these data cannot be explained by incoherent scatter. Also note that the lower frequencies contribute much more to the total power in the snow data than they do to the purely incoherent scatter (also, see the end of appendix C). For comparison in Fig. 5, we show $Z(f) \Delta f$ and $\Sigma Z(f) \Delta f$ for some rain observations. Note the much more important $20-100-\mathrm{Hz}$ contributions in rain as opposed to the $1-50-\mathrm{Hz}$ contributions in snow. Also notice that, at larger frequencies, the power rapidly drops to the white noise level. The fractional coherent contribution to the total power $\mathfrak{F}$ is estimated as discussed in appendix C. Moreover, lower bound values of $\mathfrak{F}$ can be computed also as discussed in appendix C. For these two examples, the lower bounds of $\mathfrak{F}$ corresponding to the data in Fig. 6 are 0.64 and 0.46 in snow and rain, respectively. It is not surprising, then, that the actual values in Fig. 6 are even larger.

Although the results thus far certainly indicate the presence of coherent scatter, further evidence is offered next. It comes from the pervasiveness of coherent scatter in both types of precipitation; that is, rather than just looking at individual cases, the analysis method just described can be applied to all the available rain and snow data. First, one must remember that we are only looking at $2 \mathrm{~min}$ of data albeit over several range bins. Hence, these findings should be viewed with that in mind. Using $T$ for separating coherent from incoherent fluctuations in the normalized $Z(f)$, Fig. 7 shows that coherent scatter is ubiquitous in these observations (for a demonstration showing that these results are not overly dependent on the selected threshold, see the equivalent plot of the lower bound histograms in Fig. C3). We also remind the reader that, as Fig. 3 suggests, these estimates are likely conservative.

We note that, with the exception of about $11 \%$ of the rain data, none of the radar backscatter is purely incoherent. Furthermore, there appear to be substantial differences between the snow and rain observations (in both Figs. 7a,b) perhaps reflecting different responses of the two types of hydrometeors to eddies in the wind. Thus, the examples discussed earlier are not exceptional. 

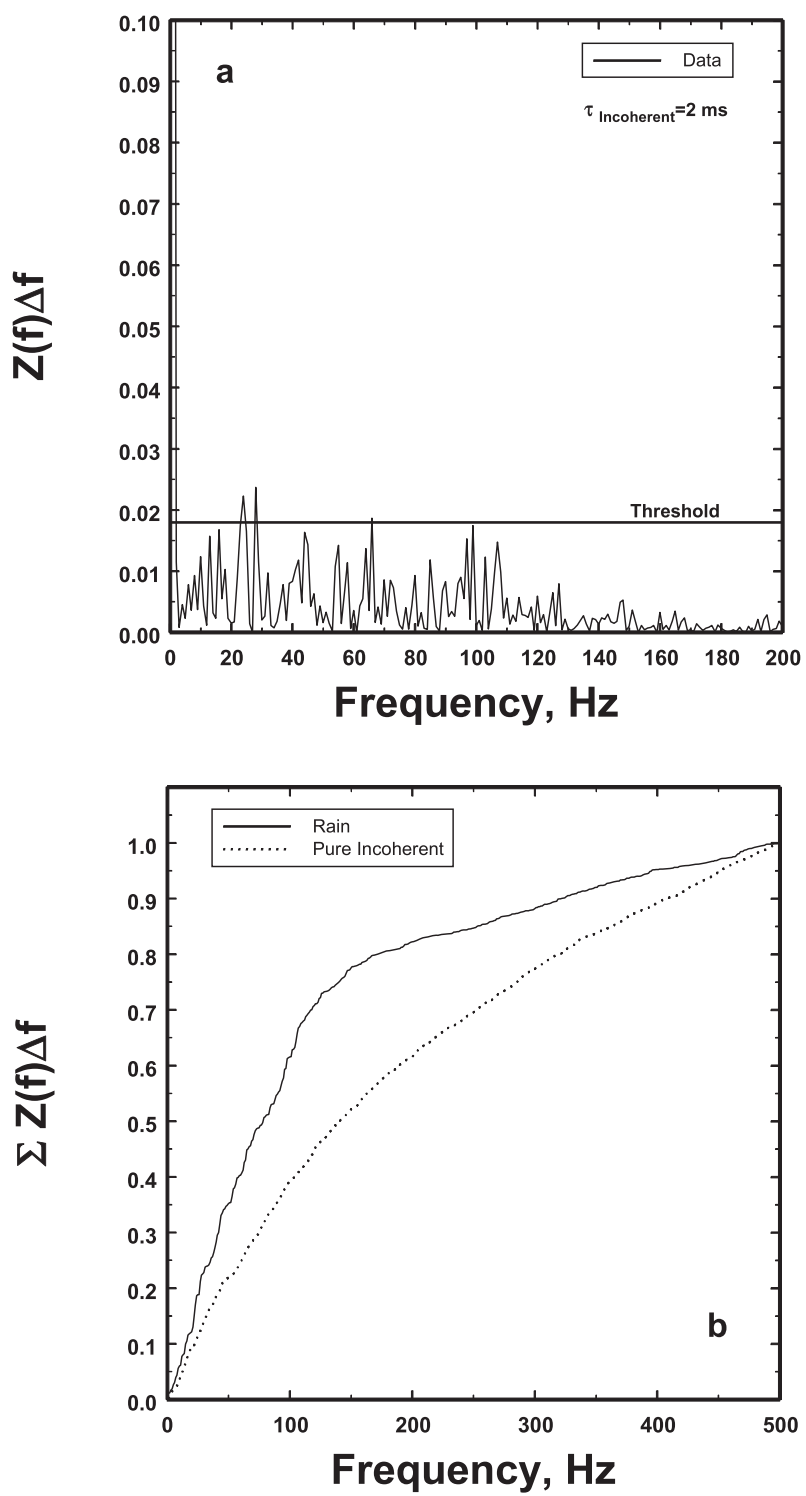

FIG. 5. (a) The power spectrum $Z(f)$ of oscillations in the radar backscattered power (obviously related to those in the amplitude) for a case in rain at RB 211. (b) A plot of the accumulated power spectrum corresponding to (a) for the data and for purely incoherent scatter simulated using the decorrelation time calculated from the observed standard deviation of the Doppler velocities. Compared to the snow case, note that much of the difference occurs between 20 and $100 \mathrm{~Hz}$ (see also Fig. C2).

In addition, radar meteorologists have often noticed the textural differences in the displays of the echoes from snow and those from rain with the former often appearing noticeably "fuzzier." Although some of this difference arises simply from the different dynamic ranges used to display snow and rain data, the results in Fig. 7 suggest that, at least in part, this difference may also be attributed to the greater frequency of what may be called "coherent speckle" in snow as compared to rain.

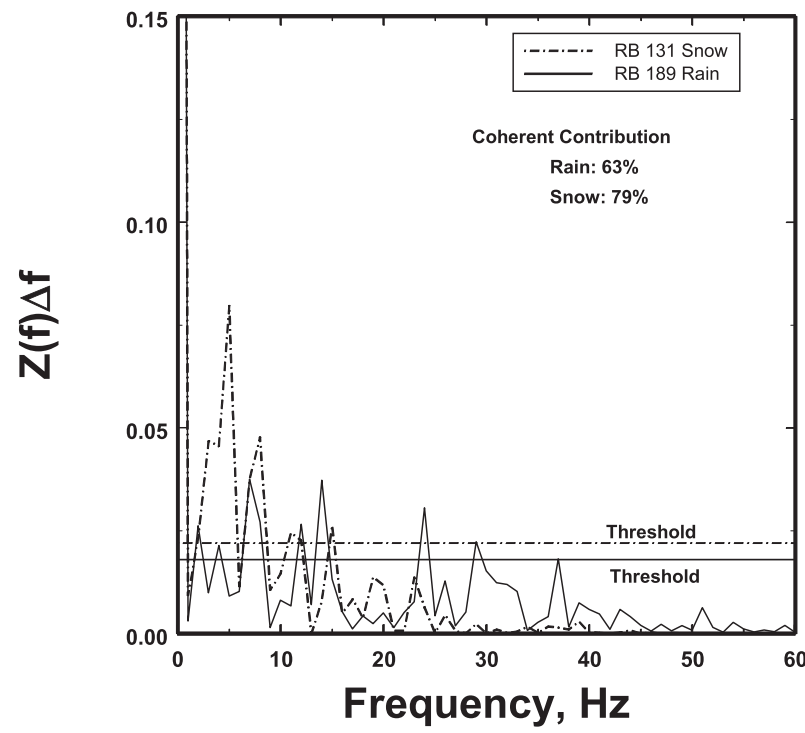

FIG. 6. Two examples of the radar backscatter power spectrum $Z(f)$ for observations in snow (dashed-dotted lines) and rain (solid lines) at the indicated RBs. The coherent contributions are found over different frequencies, as noted previously.

Finally, we consider one other piece of evidence for connecting periodic clustering of hydrometeors to radar coherent scatter by considering a serendipitous experiment as a jet airliner from the Denver International Airport passed though one of our sampling volumes. Specifically, we focus on the data over a 90-s interval in range bin $311(49.35 \mathrm{~km}$ from the radar with a sample volume of $8.74 \times 10^{7} \mathrm{~m}^{3}$ ) from the rain data described earlier. These measurements were in convective rain.

It is well known that, aside from other perturbations, aircraft produce vortices emanating from their wing tips: the larger the aircraft, the more intense and large the vortices, which can extend $10 \mathrm{~km}$ or more behind the aircraft (e.g., Kelly and Handelsman 1974; Civil Aviation Authority of New Zealand 2008). Figure 8 is an example of the effect on clouds of the vortices found behind even a small Lear jet. Although the vortices stir the clouds, such stirring does not lead to greater spatial uniformity [see also, Myers et al. (1999) for a study of Bragg scatter off of aircraft wake vortices of the atmospheric water vapor]. Rather there is increased clustering in regions of lower vorticity and higher strain rates as many investigators have noticed studying the stirring of inertial particles (analogous to raindrops in the wind) in fluids (e.g., Squires and Eaton 1991). Furthermore, airliners with their larger and more intense wake vortices are likely to influence rain over a considerable distance behind their paths through an entire radar sample volume. The central question becomes, then, do these wake vortices and other perturbations generate new periodic 

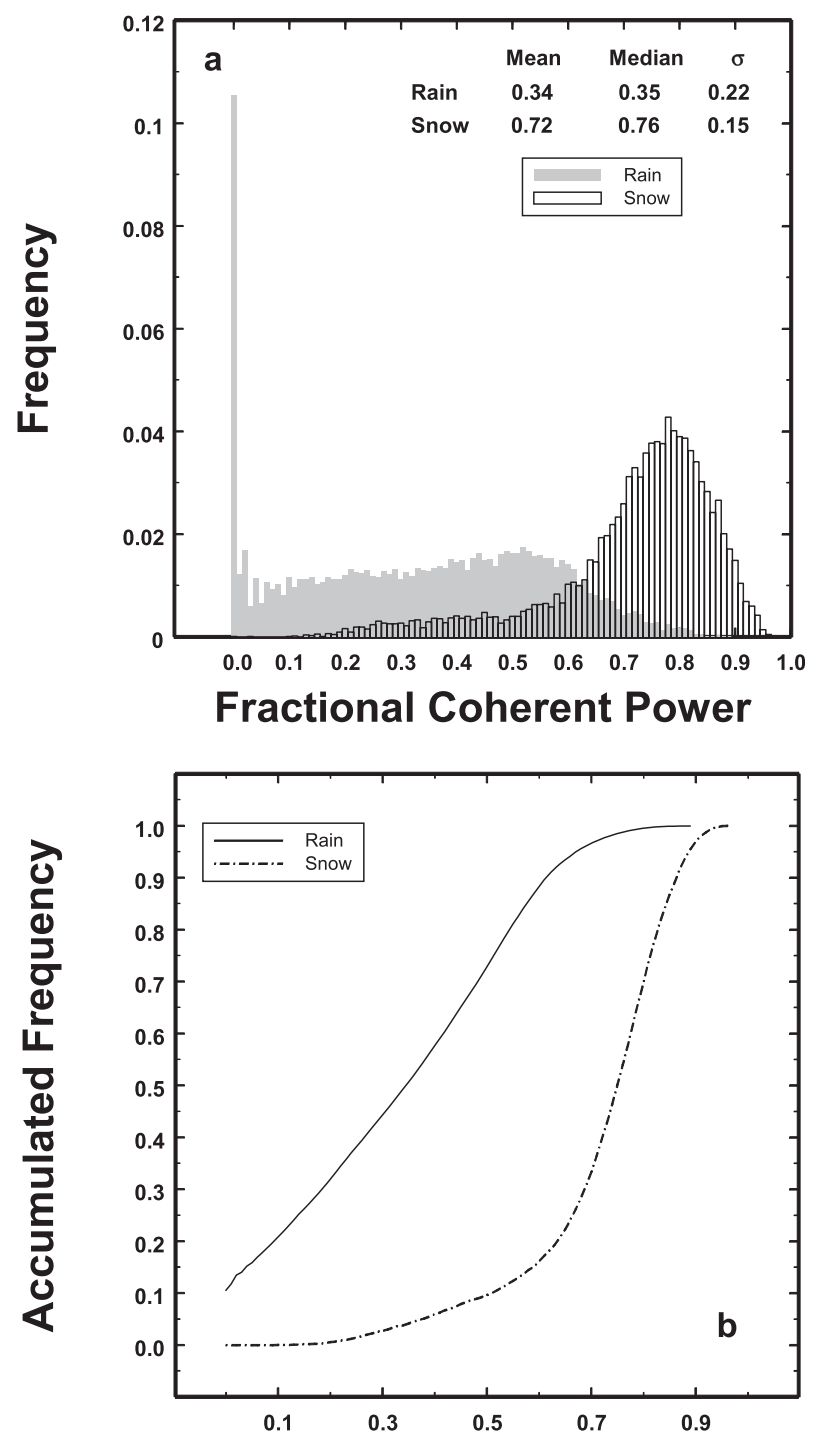

Fractional Coherent Scatter

FIG. 7. (a) The histograms of the estimated fractional coherent scatter contribution to the total backscattered power in approximately 15600 and 10400 one-second samples in rain and snow, respectively, using the thresholds as discussed in appendix C. (b) A plot of the accumulated frequencies of the fractional coherent contribution to the total backscattered power for rain and snow corresponding to (a). These and the plots in Fig. C3 suggest that coherent scatter is quite prevalent and significant in these $2 \mathrm{~min}$ of observations.

clustering in rain capable of increasing radar coherent backscatter via the mechanism just discussed?

Figure 9 illustrates the passage of the aircraft through range bin 311 captured in both the main, first, and possibly second side lobes of the radar beam as well. The largest shaded region denotes when the aircraft could be seen in the Doppler spectrum over a period of $23 \mathrm{~s}$. The inner dashed region denotes $7 \mathrm{~s}$ of missing data during

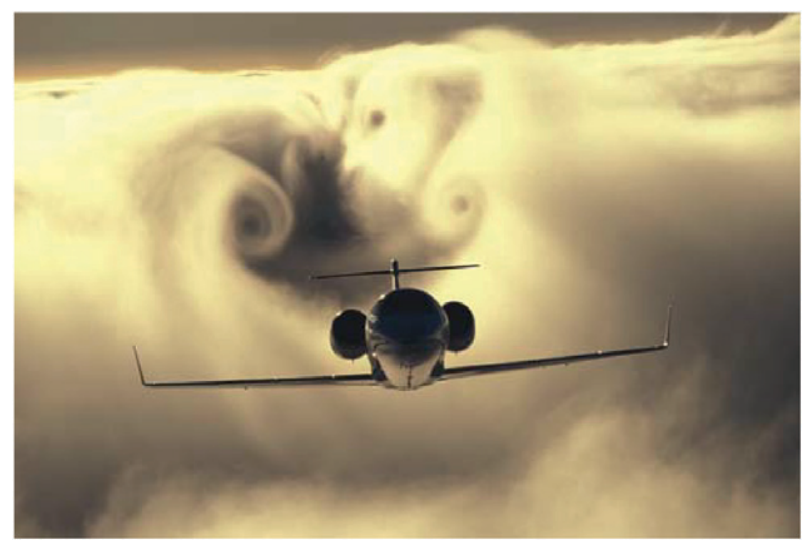

FIG. 8. Wake vortices in clouds produced by a Lear jet. Note the enhanced clustering within the vortices particularly on the left. Heavier jets produce much larger, more intense wake vortices that can extend $10 \mathrm{~km}$ and that are capable of disturbing precipitationsized particles (picture by permission of Paul Bowen; available online at http://www.airtoair.net).

a transition between two datasets. In addition we plot the observed decorrelation times of the complex amplitudes. Although one would normally expect that an aircraft would increase the stirring of the rain and that this would lead to a decrease in the decorrelation time, what we see is just the opposite. How can this be?

Although one cannot completely rule out pure coincidence, our interpretation is that the passage of the aircraft led to enhanced periodic clustering of the rain and consequently not only to the enhancement of the coherent scatter as indicated by the calculated $\mathfrak{F}$ in Fig. 9 but also to the increased decorrelation times. This is easy to imagine when the airplane is in the rain. Because of the coherency of the aircraft, the decorrelation time can greatly increase the more the aircraft dominates the signal. When there is no airplane, however, a similar phenomenon can occur by having two groups of scatterers, one with a short decorrelation time and one with a longer one, both weighted equally, say, in power, in the sample volume. The net decorrelation time will be longer than for the shorter and shorter than for the longer. Thus, we attribute the enhanced decorrelation times in Fig. 1 to the presence of coherent scatter, which extended the decorrelation times well beyond those anticipated for purely incoherent scatter. Similarly, the increase in the decorrelation time in Fig. 9 is consistent with the appearance of increased coherent scatter.

We think that the increases in Fig. 9 are significant, even though the aircraft only influences a small portion of the total sample volume. At the range observed, the main lobe radar beam was about $860 \mathrm{~m}$ in diameter. An aircraft such as a Boeing 757 leaves two wake vortices, each about $30 \mathrm{~m}$ in diameter. This means that at a minimum 


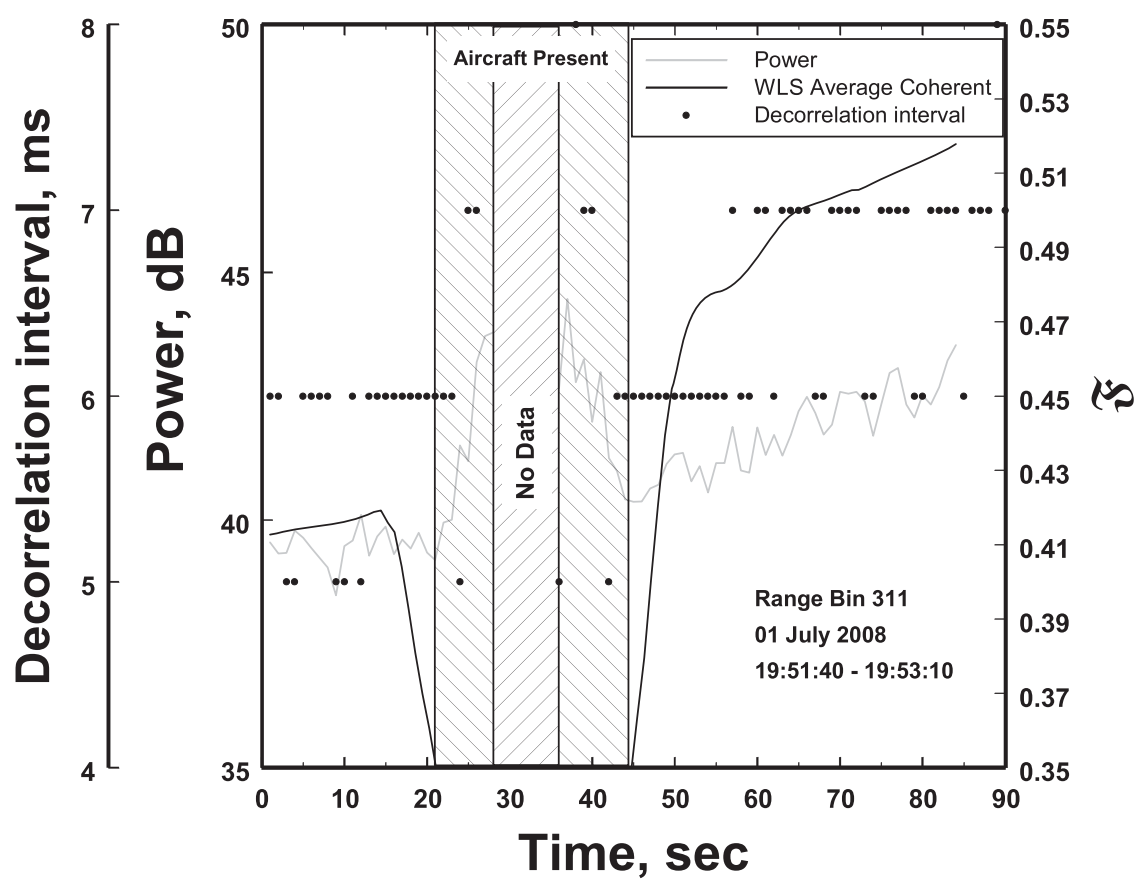

FIG. 9. Profiles of the radar backscattered power, decorrelation times, and calculated least squared weighted average fractional coherent scatter contribution to the total power $\mathfrak{F}$ for RB 311 , as discussed in the text. The increases in $\mathfrak{F}$ and $\tau$ after the passage of the aircraft are on the order of 0.1 .

the vortices could influence a volume on the order of about $1-2 \times 10^{6} \mathrm{~m}^{3}$ or about $1 \%-2 \%$ of the total sample volume in this case. Of course, an aircraft can leave other trailing vortices as well. However, although the affected volume is likely small, remember that coherent power goes as $\bar{N}^{2}$ (see appendix A) so that an aircraft can still generate a detectable effect even in a large volume of incoherent scatterers. In this case, the coherent scatter contribution increased by about $10 \%$, which is consistent with the likely small portion of the sample volume actually involved.

The presence of an aircraft also serves to illustrate the subtle nature of radar coherent scatter. In Fig. 10a, it is easy to pick out the aircraft in the Doppler spectrum as separate from the rain signals. However, when one looks at a scatter diagram of the $I$ and $Q$ channels (Fig. 10b), the distribution of points appears to be a 2D Gaussian just as though the scatter was entirely from incoherent scatterers. Although it is well known that, a fixed coherent target embedded in incoherent scatterers is described by the Rice probability distribution (Rice 1945) such that the 2D Gaussian would be displaced from the origin, which does not happen here. The subtle difference is that even though the airplane is a coherent scatterer in the sense defined by Battan (1973), as discussed in the opening of this work, the aircraft moves and this washes out any net displacement of the origin from $(0,0)$. The same is also true for radar coherent backscatter by precipitation. The intermittent grids move so that using the $I$ and $Q$ values are of no help in identifying coherent backscatter; that is, the distributions of $I$ and $Q$ are not Ricean but instead remain Gaussian as though there was no coherent scatter even when, in this case, an airplane is clearly detected.

Finally, it is important to remember that temporal and spatial coherency alone is symptomatic but not sufficient to demonstrate coherency. However, in appendix B, the association among Doppler spectral power peaks (reflecting the nearly constant velocities and the presence of periodic spatial structures in resonance with the wavelength as required in appendix A), the peak frequencies in $Z(f)$ and their association with integer multiples of $\lambda / 2$, where $\lambda$ is the radar wavelength, is illustrated. It is shown that the major peaks in $Z(f)$ associated with coherent scatter are directly related to the peaks of the Doppler velocity spectrum through integer multiples of half of the radar wavelength, as required for Bragg scatter. The spacing in the grids in this example apparently range from $1.045 \mathrm{~m}$ down to $16.5 \mathrm{~cm}$ in this snow example and from $1.5 \mathrm{~m}$ down to $38 \mathrm{~cm}$ in the rain example in appendix B. These are the dimensions projected along the beam axis so that the actual spacing is likely to be larger. 

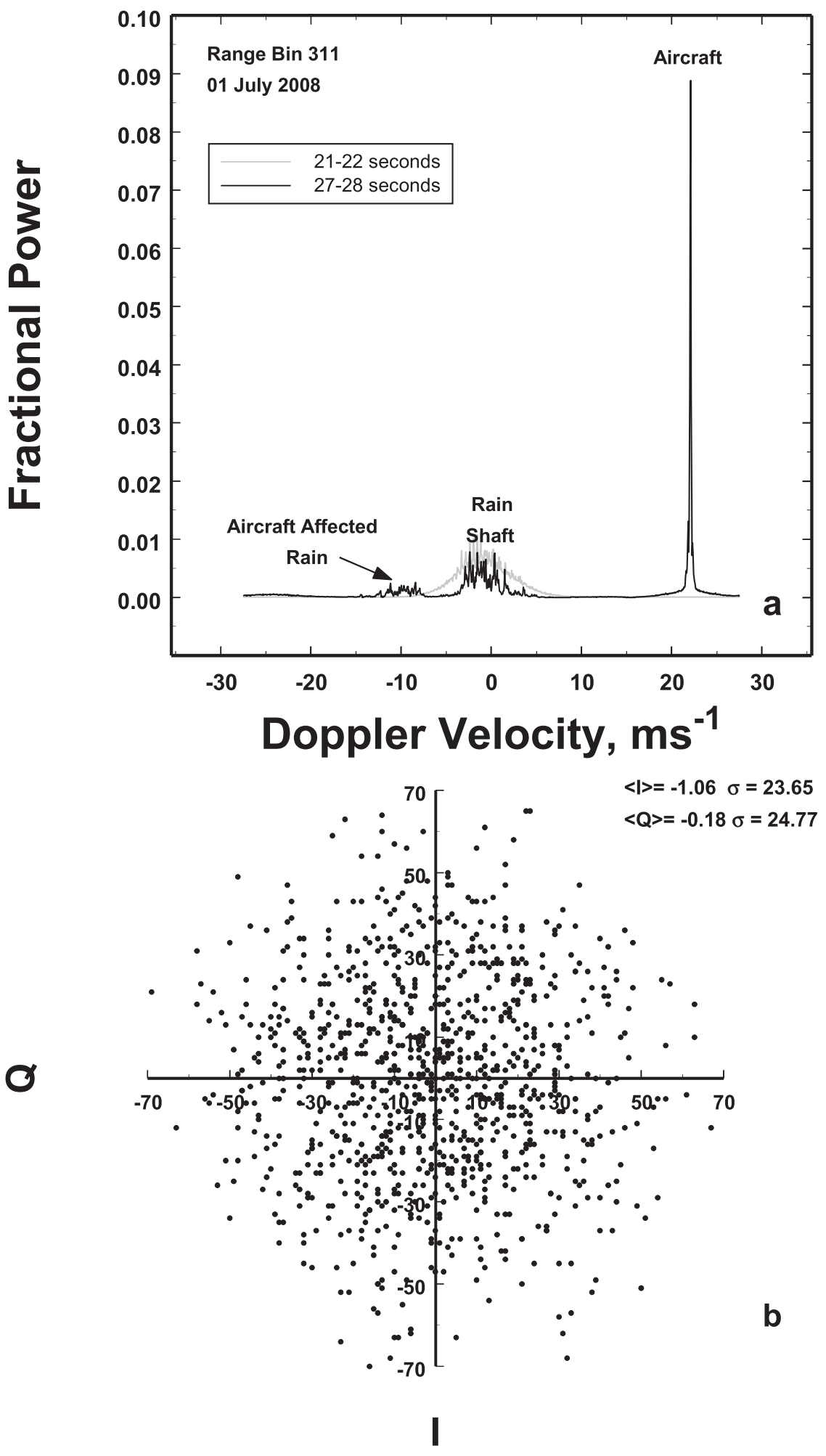

FIG. 10. (a) The Doppler spectrum containing both rain and aircraft signals. The gray curve is an additional plot of the rain signal just before aircraft penetration. (b) The scatter diagram of $I$ and $Q$ pairs corresponding to the Doppler spectrum in (a) containing the aircraft signal. There is no statistically significant displacement of the mean values from $(0,0)$, that is, the signals remain Gaussian. 


\section{Discussion}

This work presents a mechanism for the generation of radar coherent scatter in precipitation. The theory supporting this mechanism shows that the coherent scatter can be generated by spatially correlated precipitation structures acting like diffraction gratings in resonance with the radar wavelength and with elements of the structures all moving at the same velocity. Such diffraction gratings are well known to produce fields of maxima and minima of backscattered intensities. As they move through the radar beam, these fields produce distinctive power oscillations of frequencies $f$ that cannot be attributed to incoherent scatter (i.e., to velocity fluctuations).

A new backscattered power spectrum $Z(f)$ is then defined. This power spectrum is the distribution of the total backscattered power over the power oscillations characterized by $f$. [Classically, for pure incoherent scatter, $Z(f)$ is similar to the fluctuation spectrum arising from differential velocities; however, in our case $Z(f)$ is more general, because it includes additional non-Doppler fluctuations from coherent scatter.] This spectrum allows us to estimate the coherent scatter contribution to the total backscattered power. It also has the advantage that it can be computed even for incoherent radars, because Doppler information is not required.

We also note that, when coherent scatter is present, the usual relations between the standard deviation of the Doppler velocity and decorrelation time in common use are no longer valid. This is important, because it is normally assumed that the decorrelation time is inversely proportional to the standard deviation of the velocity spectrum. When coherent scatter is present, however, this is no longer true, because the signal temporal coherency is enhanced by the presence of wave coherent scatter sometimes well beyond that reflecting normal particle reshuffling as discussed at the end of section 2. Because the backscattered power consists of the coherent and incoherent components, the normal particle reshuffling times characteristic of the incoherent component can then only be estimated using Doppler observations.

Data in snow and rain were analyzed. Coherent scatter was found to be pervasive throughout these $2 \mathrm{~min}$ of data with only up to at most $11 \%$ of the rain observations being examples of pure incoherent scatter. Because coherent scatter appeared to be more pervasive in snow than in rain, the "fuzzier" appearance of radar echoes of snow compared to the radar echoes of rain may, in part, be due to the greater frequency of "coherent speckle" in the snow. This may be due to the enhanced responsiveness of the lighter snow to turbulent eddies (Kolmogorov 1941a,b), which leads to increased concentration of particles in regions of high strain and low vorticity (Squires and Eaton 1991).
Although these analyses are consistent with this mechanism, we also wanted to see if we could further characterize these diffraction gratings and explicitly detect resonance with the radar wavelength. This is done in appendix B for examples from snow and rain. There are many other examples as well, which cannot be presented in the space allotted to this work. Hence, we conclude that partially coherent backscatter is frequently present in these data.

To summarize, then, it has been shown that removing the significant $f \mathrm{~s}$ from $Z(f)$ at lower frequencies greatly reduces the temporal coherency (Fig. B1c). Thus, these lower frequencies are associated with the increased temporal coherency. It has also been shown that these $f \mathrm{~s}$ are also directly linked to the Doppler spectral peaks through integer multiples of $\lambda / 2$, characteristic of Bragg scatter (Fig. B2). Thus, we conclude that the enhanced temporal coherency can be directly linked to the presence of wave coherent scatter in agreement with the findings in appendix A. Indeed, recent research to appear in a subsequent paper (because there are so many appendices in this article already) will show that the presence of wave coherent scatter is directly related to the increased temporal coherency. Moreover, a separate paper currently in review provides a direct calculation of such coherent scatter so that we know it exists.

It seems, then, that radar coherent scatter from precipitation exists and is generated by the mechanism introduced here. Moreover, it appears to be pervasive in these data. However, because this only represents a few minutes of observations, one should not overgeneralize. Much work remains to be done if we are to fully explore the extent and statistical characterization of radar coherent scatter. The potential application of this approach to observations in clouds also needs exploration. At a minimum, though, these findings present a challenge to the assumption that the scatter of radar waves from precipitation is always incoherent; if prevalent, these findings will also require the reevaluation of many current approaches toward the quantitative interpretation of radar observations of precipitation.

Acknowledgments. This work was supported by the National Science Foundation (NSF) under Grants ATM05-31996 (AJ), ATM08-04440, and ATM05-54670 (AK). We gratefully acknowledge the meticulous data acquisition by Dave Brunkow and Pat Kennedy of the NSF CSU-CHILL National Weather Radar Facility operated by the Colorado State University (CSU).

\section{APPENDIX A}

\section{Bragg Scatter from Distributed Scatterers}

Gossard and Strauch (1983) derived the first expression for coherent Bragg scatter from particles, so 
why do it over? There are several justifications. First and foremost, this derivation includes the presence of Doppler velocity not considered before. This turns out to be very important with regard to coherent scatter by precipitation. Second, this derivation comes from a more statistical-physical approach that incorporates the pair correlation function in a very natural manner. Finally, this derivation serves to emphasize that the radar backscattered signal consists of two components, the so-called incoherent part and a second term that is usually discarded in classical treatments of the radar backscatter from particles. We show here that discarding it is not always appropriate.

The net electric field at a location produced by spatially distributed scatterers can be expressed as

$$
E=\sum_{i} a_{i} e^{j\left(\omega_{i} t-2 \mathbf{k} \cdot \mathbf{r}_{i}\right)},
$$

where $a_{i}$ is the amplitude of the field scattered by the $i$ th particle at location $\mathbf{r}_{i}$ from the observer, $\omega_{i}$ is its Doppler angular frequency, and $\mathbf{k}$ is the wavenumber along the direction of propagation and the factor of two accounts for a round trip. The instantaneous intensity $I$ is then given by the complex product $E E^{*}$ so that

$$
\begin{aligned}
I=E E^{*}= & \sum_{i} \sum_{m} a_{i} a_{m} \exp \left[j 2 \mathbf{k} \cdot\left(\mathbf{r}_{i}-\mathbf{r}_{m}\right)\right] \\
& \times \exp \left[j\left(\omega_{i}-\omega_{j}\right) t\right] .
\end{aligned}
$$

However, this sum consists of two components: namely, when $i=m$ and when $i \neq m$. Furthermore, when $i \neq m$, the summation can be separated into another two components: namely, one in which $\omega_{i}=\omega_{m}$ and one in which $\omega_{i} \neq \omega_{m}$. Consequently, we then have

$\langle I\rangle=\left\langle\sum_{i} a_{i}^{2}\right\rangle+\left\langle\sum_{i} \sum_{m \neq i} a_{i} a_{m} \exp \left[j 2 \mathbf{k} \cdot\left(\mathbf{r}_{i}-\mathbf{r}_{m}\right)\right]\right\rangle_{\omega_{i}=\omega_{m}}+\left\langle\sum_{i} \sum_{m \neq i} a_{i} a_{m} \exp \left[j\left(\omega_{i}-\omega_{m}\right) t+2 \mathbf{k} \cdot\left(\mathbf{r}_{i}-\mathbf{r}_{m}\right)\right]\right\rangle_{\omega_{i} \neq \omega_{m}}$.

where $\langle\cdot\rangle$ represents the time average over an ensemble of observations. However, because of the differential angular frequencies, the last term rapidly (on the order of $50 \mathrm{~ms}$ for a stationary antenna) goes to zero so that (A3) becomes

$$
\langle I\rangle=\left\langle\sum_{i} a_{i}^{2}\right\rangle+\left\langle\sum_{i} \sum_{m \neq i} a_{i} a_{m} \exp \left[j 2 \mathbf{k} \cdot\left(\mathbf{r}_{i}-\mathbf{r}_{m}\right)\right]\right\rangle .
$$

If we collect a time series of observations in which the particles are allowed to reshuffle a sufficient number of times, the first term will converge toward the mean $\bar{N} a^{2}$, where $\bar{N}$ is the mean number of particles in the sample volume $V$. This represents the usual incoherent scatter component of the intensity.

The second term is interesting because it represents the interference among the waves scattered by the different particles and is independent of particle reshuffling decorrelation. Because the cross sections and positions are assumed to be independent, the second term can be reexpressed as

$$
\begin{aligned}
& \left\langle\sum_{i} \sum_{m \neq i} a_{i} a_{m} \exp \left[j 2 \mathbf{k} \cdot\left(\mathbf{r}_{i}-\mathbf{r}_{m}\right)\right]\right\rangle \\
& =\overline{a^{2}}\left\langle\sum_{i} \sum_{m \neq i} \exp \left[j 2 \mathbf{k} \cdot\left(\mathbf{r}_{i}-\mathbf{r}_{m}\right)\right]\right\rangle .
\end{aligned}
$$

Now let us define $\mathbf{I}_{m}=\mathbf{r}_{m}-\mathbf{r}_{i}$. Furthermore, in order to progress, let us assume statistical homogeneity in the direction of propagation and consider elemental volume $d V$ located at $\left(l_{m}, \varphi, \theta\right)$ from the $i$ th particle such that they contain either one or no particles. The probability that an elemental volume located distance $l$ away from the $i$ th particle contains a particle is given by

$$
P_{m}=\frac{\bar{N} d V}{V}[1+\eta(l)]=\bar{n}[1+\eta(l)] d V,
$$

where $\bar{N}$ is the total mean number of particles in sample volume $V$ and $\eta(l)$ is the pair correlation function. Now, because either $d V$ contains a particle or not, integrating $P_{m}$ over $V$ will allow us to evaluate the second summation in (A4), which now becomes

$$
\begin{aligned}
& \langle I\rangle=\overline{a^{2}}\left\langle\frac{\bar{N}}{V} \sum_{i} \sum_{m \neq i}\left[1+\eta\left(l_{m}\right)\right] \exp \left[-j 2 \mathbf{k} \cdot \mathbf{I}_{m}\right] d V_{m}\right\rangle \\
& \langle I\rangle=\overline{a^{2}} \frac{\bar{N}}{V}\left\langle\bar{N} \sum_{m \neq i} \exp \left(-j 2 \mathbf{k} \cdot \mathbf{I}_{m}\right) d V_{m}+\bar{N} \sum_{m \neq i} \eta\left(l_{m}\right) \exp \left(-j 2 \mathbf{k} \cdot \mathbf{I}_{m}\right) d V_{m}\right\rangle \text { or }
\end{aligned}
$$




$$
\langle I\rangle=\overline{a^{2}} \frac{\bar{N}^{2}}{V}\left\langle\left[\sum_{m \neq i} \exp \left(-j 2 \mathbf{k} \cdot \mathbf{I}_{m}\right) d V_{m}+\sum_{m \neq i} \eta\left(l_{m}\right) \exp \left(-j 2 \mathbf{k} \cdot \mathbf{l}_{m}\right) d V_{m}\right]\right\rangle .
$$

Now consider the case when $\eta(l)$ is identically zero everywhere. Then (A8) reduces to

$$
\langle I\rangle=\overline{a^{2}} \frac{\bar{N}^{2}}{V}\left\langle\sum_{m \neq 1} \exp \left(-j 2 \mathbf{k} \cdot \mathbf{I}_{m}\right) d V_{m}\right\rangle .
$$

Because there is no correlation (since $\eta(l)=0$ on all scales), all the contributions are statistically independent, and we may bring the bracket within the summation: that is,

$$
\langle I\rangle=\overline{a^{2}} \frac{\bar{N}^{2}}{V} \sum_{i \neq i}\left\langle\exp \left(-j 2 \mathbf{k} \cdot \mathbf{1}_{m}\right)\right\rangle d V_{m} .
$$

We now note that the expected value of each exponential will be zero. We then note that, because this term is the same regardless of $\eta(l)$, it must always be zero. Consequently, we are then left with the second term in (A8). However, in this case the brackets cannot be brought within the summation because of $\eta(l)$. This term, therefore, will exhibit coherency in a time series because of the spatial structure of the scatterers. We can, therefore, identify this as the Bragg scatter component of the intensity. We focus exclusively on this term later.

Now because $d V$ is so small that it contains either one or no drops, we can replace the second summation in (A8) with integrals over the sample volume $V$ so that

$$
\left\langle I_{B}\right\rangle=\overline{a^{2}} \frac{\bar{N}^{2}}{V}\left\langle\int_{V} \eta(l) \exp \left(-j 2 \mathbf{k} \cdot \mathbf{I}_{m}\right) d V\right\rangle
$$

The integral can be computed as follows: Using our spherical coordinates, we can now write

$$
d v=l^{2} d l \sin \theta d \theta d \varphi
$$

where $\varphi$ is the orthogonal angle to $\theta$, the angle between $\mathbf{k}$ and $\mathbf{l}$. Because $\mathbf{k} \cdot \mathbf{l}=k l \cos \theta$ and because the particles can assume all relative angles while $l$ can go from 0 to very large values, the equation can be rewritten to become

$$
\left\langle I_{B}\right\rangle=\frac{\bar{N}^{2}}{V} \overline{a^{2}}\left\langle\int_{0}^{\infty} l^{2} \eta(l) d l \int_{0}^{2 \pi} \int_{-\pi}^{\pi} \exp (-2 j k l \cos \theta) \sin \theta d \theta d \varphi\right\rangle .
$$

Now

$$
\begin{aligned}
& \int_{-\pi}^{\pi} \exp (-j 2 k l \cos \theta) \sin \theta d \theta \\
& \quad=\int_{-1}^{1} \exp (-j 2 k x) d x=\frac{1}{k l} \sin (-2 k l)
\end{aligned}
$$

so that

$$
\begin{aligned}
& \left\langle I_{B}\right\rangle=\frac{\bar{N}^{2} \overline{a^{2}} 2 \pi}{V k}\left\langle\int_{0}^{\infty} \ln (l) \sin (-2 k l) d l\right\rangle \text { or } \\
& \left\langle I_{B}\right\rangle=\frac{\bar{n}^{2} V \overline{a^{2}} 2 \pi}{k}\left\langle\int_{0}^{\infty} \ln (l) \sin (-2 k l) d l\right\rangle,
\end{aligned}
$$

where $l$ is the separation distance between scatterers in the direction of the transmission. This is similar to the expression of Gossard and Strauch [1983, Eq. (2-14b)] using the substitution

$$
\eta(l)=\frac{\sigma^{2}(n)}{\bar{n}^{2}} C(l),
$$

except that we now have a better physical understanding of the origin of the relation and the role that Doppler velocity plays.

In particular, the brackets in (A16) denote a temporal average. This arises because the elements of these structures all move at the same Doppler velocity so that they are not rapidly torn apart by particle reshuffling. However, they then act like diffraction gratings capable of inducing oscillations in the magnitudes of the backscattered radar amplitudes at frequencies less than those destroyed by particle reshuffling as discussed further in the text. Although a time dependence in (A16) is not explicit, it is implicit because coherent scatter from the grid of particles will produce a Fraunhofer-like spatial intensity pattern. As the grid moves, the intensity will oscillate at a period $t=1 / v_{r}$, where $v_{r}$ is the radial velocity of the grid and $l$ is the apparent spacing of the grid elements, each with respect to the radar. (There can also be oscillations even when the grid only moves perpendicular to the radial as well, but then there is no way to estimate the apparent grid spacing.) Moreover, if it should happen that more than one grid occurs simultaneously, there can be beat frequency modulations to the intensity as well, although this is thought to be relatively rare given the intermittency of the grids (shown by wavelet analysis not included here) and the findings in appendix B. 

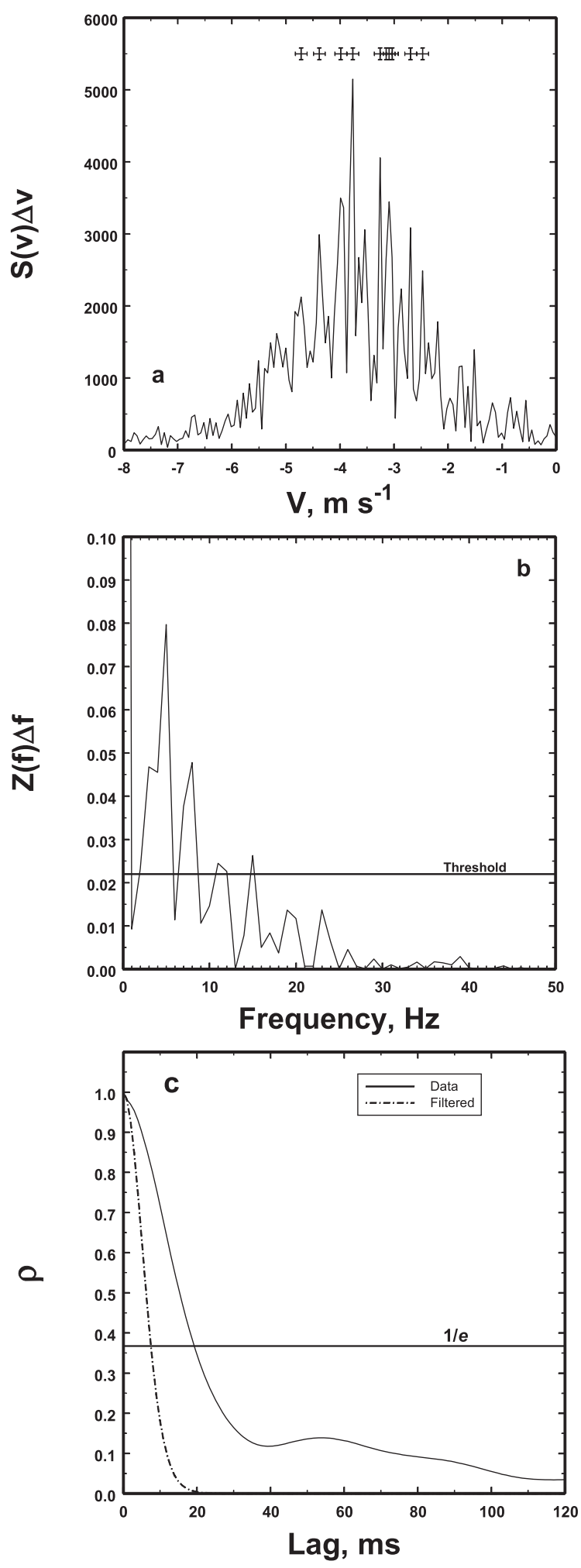

Consequently, $I_{B}$ is really $I_{B}(t)$ so that the total backscattered power is then the sum

$$
\begin{aligned}
\langle I\rangle & =\left\langle\sum_{i} a_{i}^{2}\right\rangle+\left\langle I_{B}(t)\right\rangle \\
& =\langle I\rangle_{\text {incoherent }}+\left\langle I_{B}(t)\right\rangle,
\end{aligned}
$$

The coherent power, then, comes from periodic structures of particles characterized by $\eta$ if they exist and if they are in resonance with the radar wavelength.

It is also worth repeating that these amplitude frequencies are not Doppler frequencies. This is easy to appreciate by simply considering, say, a metalized, oscillating (expanding-contracting again and again) balloon moving at some velocity. While the Doppler velocity remains constant, the radar cross section is oscillating at a frequency independent of the Doppler velocity so that this frequency oscillation will not show up as a feature in the Doppler spectrum.

\section{APPENDIX B}

\section{On the Apparent Link between Coherent Scatter and Precipitation Diffraction Gratings}

Although the backscattered power spectrum $Z(f)$ can be used to measure the coherent scatter contribution to the total backscattered power, even for an incoherent radar, to gain further insights into the characterization of the gratings and to connect the power spectral peaks to a resonance with the radar wavelength, Doppler radar observations are required. Specifically, the Doppler velocity spectrum of the coherent scatter component is computed from the difference between the complete Doppler spectrum and that corresponding to the incoherent scatter. This latter spectrum is computed by first replacing all frequencies $<1 /(2 \tau)$ in the amplitude frequency spectrum by white noise (see Fig. 2 for an example of this spectrum). The time series of the complex amplitudes for this incoherent component is then reconstructed using the phases of the original amplitudes. A normal Fourier transform is then used to yield the incoherent scatter Doppler spectrum that is then removed from the complete Doppler spectrum to yield the coherent power Doppler spectrum

FIG. B1. (a) The coherent backscatter power Doppler velocity spectrum for snow at RB 131 at 28-29 s. The significant Doppler spectral peaks are denoted by the crosses at the top of the figure. (b) The backscattered power spectrum corresponds to the data in (a). The significant peaks in $Z(f)$ are obvious and are used in Fig. B2. (c) The effect of removing the significant lower frequencies in (b) is to markedly reduce the time to decorrelation as shown in the magnitudes of the autocorrelation function of the amplitudes $\rho$. 


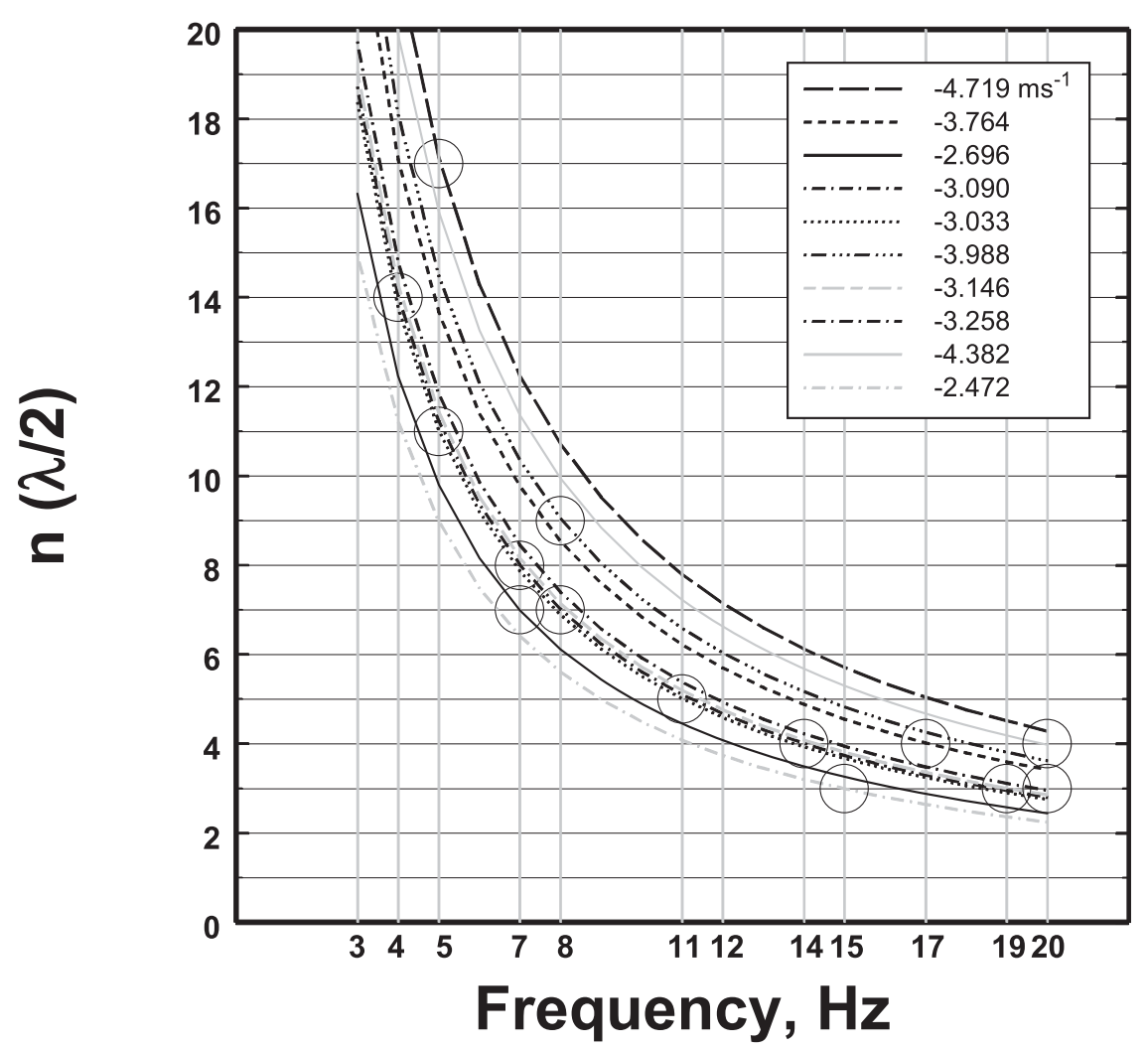

FIG. B2. A plot of the number of radar half-wavelengths as a function of the backscattered coherent power spectral peaks in Fig. B1b for the Doppler velocity spectral peaks in Fig. B1a. The circles denote the intersections of these velocity curves with the backscattered coherent spectral peaks. The circled values all lie to within \pm 0.02 of an integer value. These intersections occur at integer numbers of half-wavelengths as expected for Bragg scatter.

plotted in Fig. B1a. Here it is worth pointing out that, when the important lower frequencies evident in Fig. B1b are partially removed, the time to decorrelation drops from $20 \mathrm{~ms}$ to about $10 \mathrm{~ms}$ (Fig. B1c). Thus, the important frequencies in $Z(f)$ are directly linked to the enhanced temporal coherence.

The significant Doppler spectral peaks are denoted by the crosses at the top of the figure. Because the particles have to be moving at essentially the same velocity (see appendix A) and they have to be in resonance with the radar wavelength (see appendix $\mathrm{A}$ ), these power spikes should be associated with coherent scatter. To see this, let us consider $Z(f)$ given in Fig. B1b. These frequencies are then denoted by the vertical gray lines in Fig. B2. In addition, for each of the radial velocities $v_{r}$ corresponding to the peaks in Fig. B1a and for the radar wavelength $\lambda$, we calculate the number of $\lambda / 2$ from the relation $n=2 v_{r} / f \lambda$. The curves for all the significant Doppler spectral peaks are plotted in Fig. B2.

Although $n$ can assume any real or integer value, we denote the intersections of the significant frequencies of $Z(f)$ with the velocities of the Doppler spectral peaks by circles centered on these intersections. These values fall to within \pm 0.02 of the integer value (calculations show that there is less than a 1 in 100 billion probability that all of these circles would fall to within those bounds by chance alone). Because each velocity peak in the Doppler velocity can be associated with peaks in $Z(f)$ at exact integer multiples of $\lambda / 2$ (to within \pm 0.02 of the integer), it appears that the coherent scatter is likely coming from Bragg-like diffraction gratings moving in the embedding wind and producing the spectral peaks in the intensity oscillations described by $Z(f)$ with little if any from beat frequencies among simultaneous grids. Moreover, the spacing in the grids apparently range from $1.045 \mathrm{~m}$ down to $16.5 \mathrm{~cm}$. However, these are the dimensions projected along the beam axis so that the actual spacing is likely to be larger (by a factor of 1.4 if the average viewing angle is $45^{\circ}$ ).

Finally, for completeness we consider another Doppler spectrum, this time in rain (Fig. B3) where the statistically significant spectral peaks are associated with the corresponding integer multiples of the radar half-wavelength. The total spectral power is spread out over all the velocity bins but obviously not uniformly. Consequently, mean 


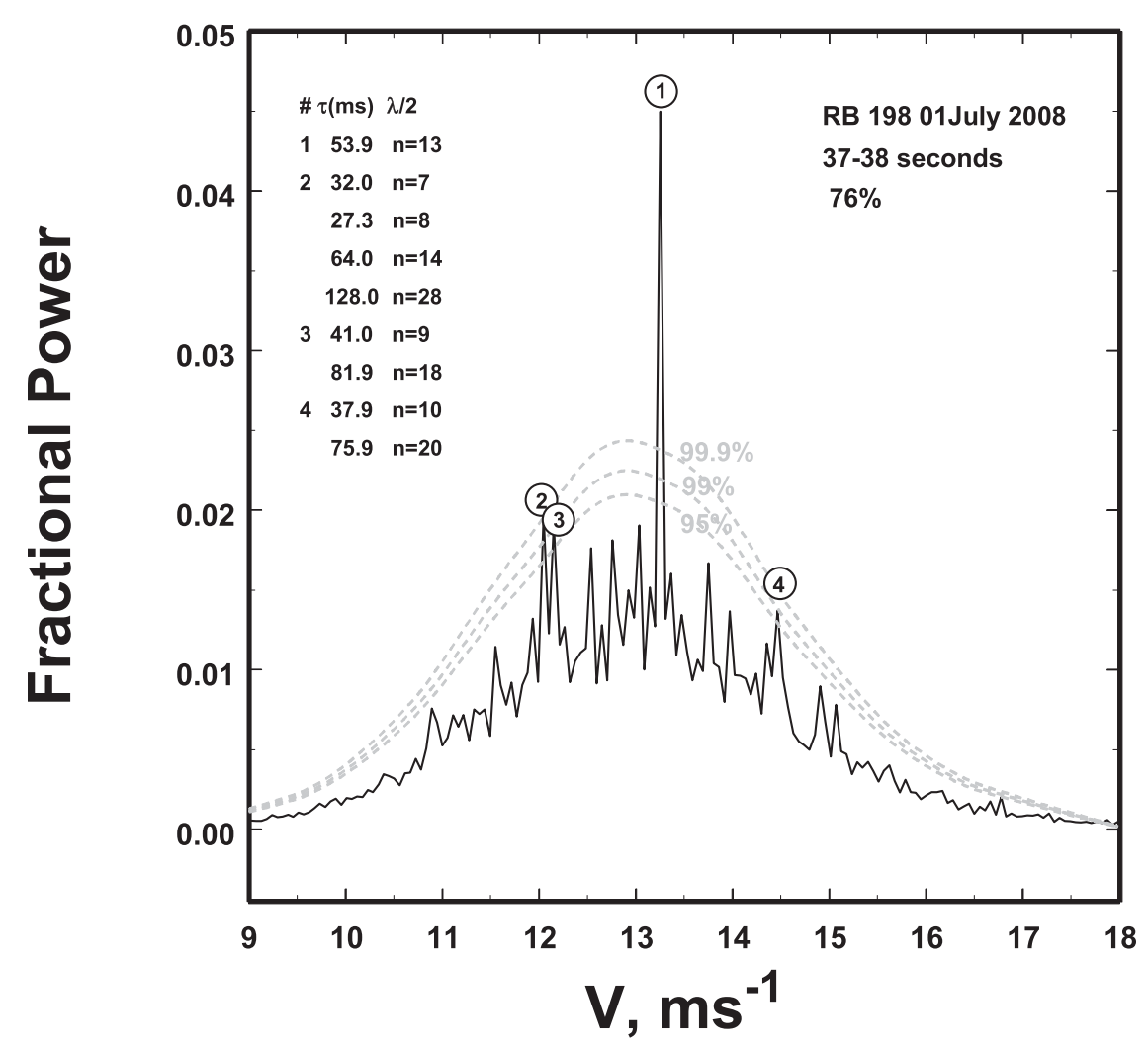

FIG. B3. The analyses of a Doppler spectrum in rain. Values of the integer number of radar half-wavelengths are indicated for the four most statistically significant spectral peaks, as discussed in the text.

spectral powers corresponding to the velocity bins were computed using a least squares error fit. Moreover, because the antenna is stationary, Rayleigh signal statistics apply to each velocity bin. Now, because the intersample interval was approximately $1 \mathrm{~ms}$, the 1000 sample spectra of these data yield, on average, one statistically independent sample per velocity bin. However, this varies, so that the average number of samples across the part of the spectra with significant power was determined using histograms of the sample to sample velocity data. In this case, there were, on average, 10 samples at the significant Doppler velocity bins. Moreover, these samples were found to be statistically independent, occurring at widely disparate times because of phase randomness. Thus, for each mean power in each velocity bin, one can compute the statistical properties for the corresponding Erlang distribution (e.g., Evans et al. 1993, 55-56) with 10 statistically independent samples. The appropriate Erlang distributions are the source of the confidence limit curves plotted in Fig. B3; that is, points found lying above a particular confidence level are likely not due to statistical fluctuations to that degree of confidence.

To summarize, then, it has been shown that removing the significant $f$ from $Z(f)$ at lower frequencies greatly reduces the temporal coherency. Thus, these lower frequencies are associated with the increased temporal coherency. It has also been shown that these $f \mathrm{~s}$ are directly linked to the Doppler spectral peaks through integer multiples of $\lambda / 2$, characteristic of Bragg scatter. Thus, the enhanced temporal coherency can be directly related to the presence of wave coherent scatter in agreement with the findings in appendix A.

\section{APPENDIX C}

\section{On the Analyses of the Data}

The analyses of these data consisted of three components: namely, the calculations, the data editing, and finally the frequency analyses. The data were the $I$ and $Q$ components of the complex amplitudes, which were then used to calculate the radar power intensities $Z$ every millisecond. To survey these data, they were all processed in blocks of $1000 \mathrm{~ms}$ to yield several parameters including the observed 1/e time to decorrelation $\tau$, the maximum fluctuation in the amplitude $M_{A}$ beyond a lag of $20 \mathrm{~ms}$ for rain and $40 \mathrm{~ms}$ for snow, and the coherent fractional contribution to the total power $\mathfrak{F}$ using a procedure 


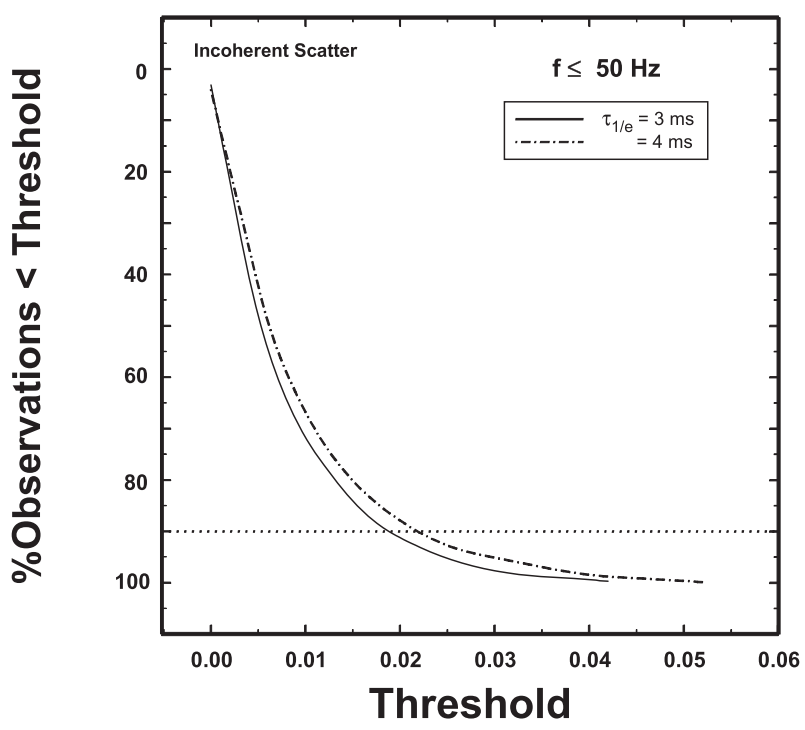

FIG. C1. A plot of the percentages of observations of $Z(f)$ at frequencies $\leq 50 \mathrm{~Hz}$ lying below a particular threshold for pure incoherent scatter estimated from an ensemble of correlated Gaussian simulations having 1/e decorrelation times characteristic of rain (3 ms) and of snow (4 ms) based on the mean values of Fig. 1. The dashed line indicates when random fluctuations of $Z(f)$ in this frequency range remain below a threshold of 0.018 in rain and 0.022 in snow $90 \%$ of the time so that at least $90 \%$ of the observed values above these thresholds likely have a physical cause. These thresholds were used in computing Fig. 7a.

described in detail here. Scatterplots of $M_{A}$ versus $\tau$ quickly revealed the domain of data unaffected by aircraft or clutter. Only data satisfying $M_{A} \leq 0.37$ and $\tau \leq 20 \mathrm{~ms}$ in rain and $40 \mathrm{~ms}$ in snow were kept. (Subsequent calculations showed that setting the upper limits even as high as 100-200 ms had little effect on the results in, e.g., Fig. 7, because of the limited occurrences of such data.) All other data were rejected. This left 26000 one-second values in the rain and snow for further analyses.

Perhaps one of the most challenging aspects of these analyses was the separation of presumed coherent from incoherent scatter fluctuations in the spectra of the intensities $Z(f)$, where $f$ is the frequency in hertz. Although for purely incoherent scatter having no sample to sample correlation there are no preferred frequencies (white noise) so that the power spectra should be flat, in reality samples are usually correlated as shown in Fig. 2. Such correlation can produce colored noise such that, in this case, the spectral magnitudes increase as the frequency decreases. This was explored using numerically generated correlated samples. For the 1000-point sample length used here, coloration increases in importance with increasing $\tau$ (i.e., increasing correlation). As Fig. 1 shows, $\tau$ corresponding to the incoherent component of the backscatter is on the order of $3 \mathrm{~ms}$ in rain and $4 \mathrm{~ms}$ in snow. Normalized (total spectral power $=1) Z(f)$ spectra were

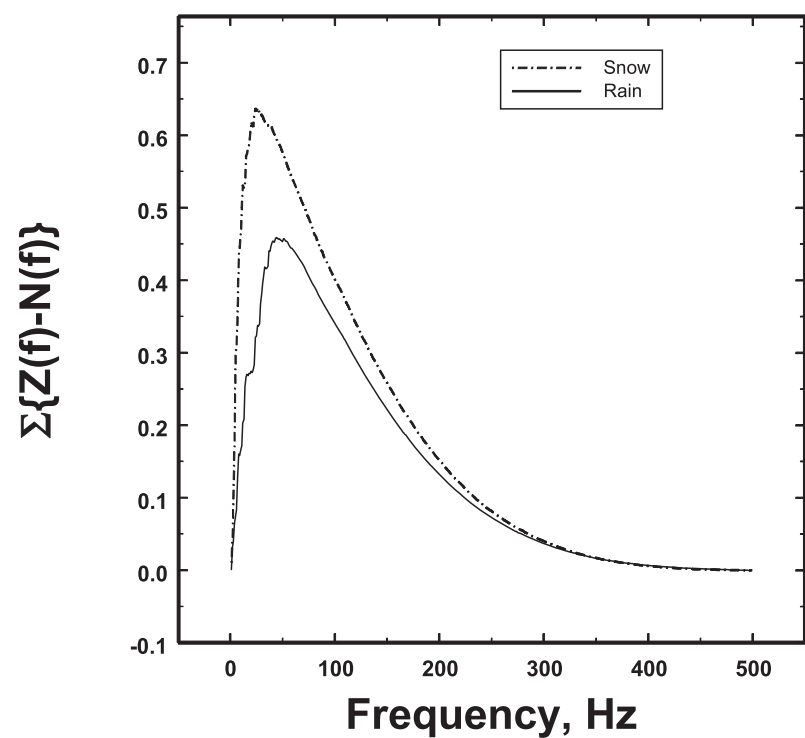

FIG. C2. Plots of the accumulated differences between the normalized spectral powers $Z(f)$ for the rain and snow examples in Fig. 6 and the average normalized spectral incoherent scatter noise $N(f)$ calculated for 50 spectral realizations having $\tau=2 \mathrm{~ms}$. As discussed in the text, each peak yields a lower bound estimate of the fractional coherent contribution to the total power $\mathfrak{F}$.

computed using 50-1000 data samples of numerically simulated correlated incoherent scatter (our "noise") at both $\tau$. From these spectra, we considered only those frequencies $\leq 50 \mathrm{~Hz}$, the band most often found to contain most of the oscillation frequencies. We then computed the percentage of times the fluctuations lay below various thresholds of $Z(f)$, as illustrated in Fig. C1. The horizontal line denotes when $90 \%$ or more of the incoherent $Z(f)$ lie below the thresholds so that at least $90 \%$ of the observed values above these thresholds likely have a physical cause. The thresholds used for rain and snow in the computations were 0.018 and 0.022 , respectively. (Although one could insist on, e.g., a 99\% exclusion, much real data would also be eliminated by too stringent a requirement.) Furthermore, as Fig. 3 suggests, these thresholds are likely conservative, because the actual $Z(f)$ arising from fluctuation velocities alone are likely much smaller than the approach here suggests.

So, then, how is the fractional coherent scatter contribution $\mathfrak{F}$ estimated? Because $Z(f)$ is a power spectrum, we can then estimate the coherent power $Z_{C}$ and the incoherent power $Z_{I}$ from

$$
\begin{aligned}
Z_{C} & =\sum_{1}^{f_{\max }} Z(f \mid Z \geq T) \quad \text { and } \\
Z_{I} & =\sum_{1}^{f_{\max }} Z(f \mid Z<T),
\end{aligned}
$$




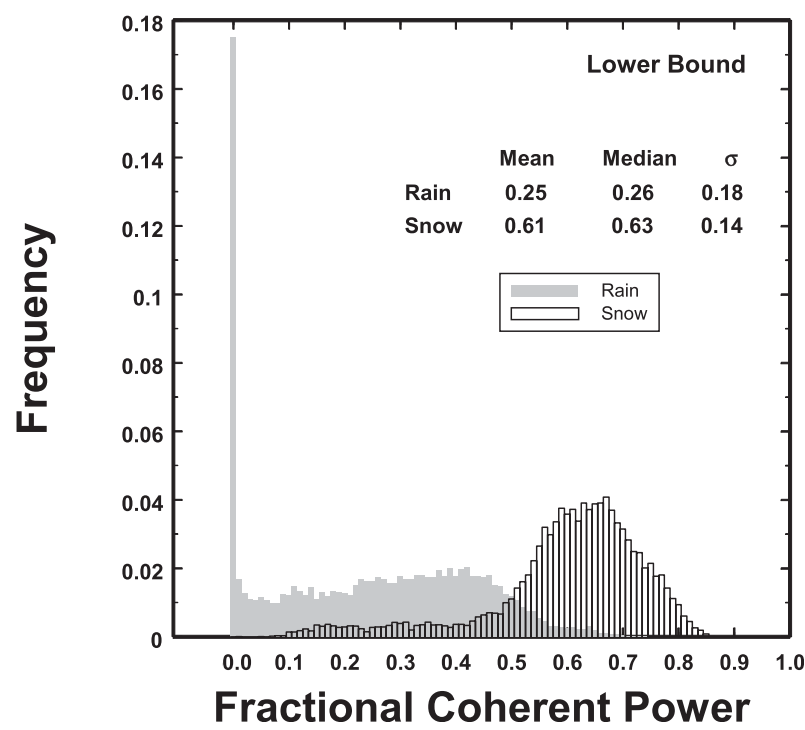

FIG. C3. Histograms of the lower bound coherent scatter contributions $\widetilde{F}_{\mathrm{LB}}$ to the total powers using the peak and mean $\tau$ of $3 \mathrm{~ms}$ for the decorrelation times in rain and $4 \mathrm{~ms}$ in snow computed from the expected values for incoherent scatter (Fig. 1a). Although shifted toward smaller values because $\mathfrak{F}_{\text {LB }}<\mathfrak{F}$, they are of quite similar form to those in Fig. 7a.

where $f_{\max }$ is the maximum spectral frequency limit and $T$ is the threshold discussed earlier [one could use the $1 /(2 t)$ frequency limit in (C1), but it turns out not to matter because the powers at higher frequencies all lie well below $T$ ]. The fractional coherent contribution is then given by the ratio $\mathfrak{s}=Z_{c} /\left(Z_{i}+Z_{c}\right)$.

It is also worth noting that the tails of the normalized $Z(f)$ are suppressed according to the magnitude of $\mathfrak{F}$. This is evident in many of the figures. For normalized spectra, $\widetilde{f}$ can be computed as

$$
\mathfrak{F}=\sum_{1}^{f_{\max }}[Z(f)-N(f)],
$$

where $N(f)$ is the spectrum of the incoherent noise. Because $Z(f)$ and $N(f)$ are both normalized, this sum over all frequencies would always be zero. However, there will be a maximum before the noise contribution starts to catch up to the coherent power contribution. This is illustrated in Fig. C2 for the two examples in Fig. 6 using the $\tau$ for incoherent scatter noise component based on the observations.

The peaks indicate that the lower bound fractional coherent power contributions $\mathfrak{F}_{\mathrm{LB}}$ in the snow and rain are 0.64 and 0.46 , respectively. The reason that these are lower bounds is simply that the equal normalized powers for both the signal and the noise are simultaneously equal to the total power. In effect, this removes any of the real difference that may exist between the total powers $Z$ and $N$. Such differences must always produce $\mathfrak{F}>\mathfrak{F}_{\mathrm{LB}}$, because in normalized spectra the signal takes power from the noise or there would be no signal.

Moreover, as a check on Fig. 7a, we also computed the histograms for these $\mathfrak{F}_{\text {LB }}$. These are shown in Fig. C3. Although there is a general shift to the left of the distributions compared to those in Fig. 7a because $\mathfrak{F}_{\mathrm{LB}}<\mathfrak{F}$, it is clear that they possess the same shapes as the distributions in Fig. 7a. Consequently, the thresholds appear adequate.

\section{REFERENCES}

Atlas, D., 1964: Advances in radar meteorology. Adv. Geophys., 10, 317-478.

Battan, L. J., 1973: Radar Observation of the Atmosphere. Techbooks, $324 \mathrm{pp}$.

Civil Aviation Authority of New Zealand, 2008: Wake turbulence. Civil Aviation Authority of New Zealand Rep., 16 pp. [Available online at www.caa.govt.nz/safety_info/gaps/wake_ turbulence.pdf.]

Erkelens, J. S., V. K. C. Venema, H. W. J. Russchenberg, and L. P. Ligthart, 2001: Coherent scattering of microwaves by particles: Evidence from clouds and smoke. J. Atmos. Sci., 58, 1091-1102.

Evans, M., N. Hastings, and B. Peacock, 1993: Statistical Distributions. 2nd ed. John Wiley and Sons, $170 \mathrm{pp}$.

Gossard, E. E., and R. G. Strauch, 1983: Radar Observations of Clear Air and Clouds. Elsevier, 280 pp.

Ishimaru, A., 1997: Wave Propagation and Scattering in Random Media. IEEE Press, 600 pp.

Jameson, A. R., and A. B. Kostinski, 1996: Non-Rayleigh signal statistics caused by relative motion during measurement. J. Appl. Meteor., 35, 1846-1859.

Kelly, A. J., and M. Handelsman, 1974: Electromagnetic effects of aircraft wake-active feuillet interaction. IEEE Trans. Aerosp. Electron. Syst., 10, 136-143.

Knight, C. A., and L. J. Miller, 1993: First radar echoes from cumulus clouds. Bull. Amer. Meteor. Soc., 74, 179-188.

Kolmogorov, A. N., 1941a: The local structure of turbulence in incompressible viscous fluid for very large Reynolds numbers (in Russian). Dokl. Akad. Nauk SSSR, 30, 299-303.

- 1941b: Dissipation of energy in locally isotropic turbulence (in Russian). Dokl. Akad. Nauk SSSR, 32, 16-18.

Kostinski, A. B., and A. R. Jameson, 2000: On the spatial distribution of cloud particles. J. Atmos. Sci., 57, 901-915.

Lhermitte, R. M., and E. Kessler, 1966: Estimation of the average intensity of precipitation targets. Proc. 12th Conf. on Radar Meteorology, Norman, OK, Amer. Meteor. Soc., 23-27.

Marshall, J. S., and W. Hitschfeld, 1953: Interpretation of the fluctuating echo from randomly distributed scatterers. Part I. Can. J. Phys., 31, 962-994.

Myers, T. J., W. A. Scales, and R. E. Marshall, 1999: Determination of aircraft wake vortex radar cross section due to coherent Bragg scatter from mixed atmospheric water vapor. Radio Sci., 34, 103-111. 
Rayleigh, J. W. S., 1945: The Theory of Sound. 2nd ed. Dover, $480 \mathrm{pp}$.

Rice, S. O., 1945: Mathematical analysis of random noise. Bell Syst. Tech. J., 24, 46-156.

Rogers, R. R., and W. O. J. Brown, 1997: Radar observations of a major industrial fire. Bull. Amer. Meteor. Soc., 78, 803-814.

Rughaven, S., 2003: Radar Meteorology. Springer, 564 pp.

Siegert, A. J. F., and H. Goldstein, 1990: Coherent and incoherent scattering from assemblies of scatterers. Propagation of Short
Radio Waves, IEE Electromagnetic Wave Series, Vol. 24, Institution of Electrical Engineers, 699-706.

Squires, K. D., and J. K. Eaton, 1991: Preferential concentration of particles by turbulence. Phys. Fluids, 3, 11691178.

Tatarskii, V. I., 1961: Wave Propagation in a Turbulent Medium. McGraw-Hill, 285 pp.

Wolf, E., 2007: Introduction to the Theory of Coherence and Polarization of Light. Cambridge University Press, $236 \mathrm{pp}$. 